\title{
Multiple Object Tracking Via Multi-layer Multi-modal Framework
}

\author{
Hang-Bong Kang and Kihong Chun \\ Dept. of Computer Eng., Catholic University of Korea \\ \#43-1 Yokkok 2-dong Wonmi-Gu, Puchon, Kyonggi-Do Korea \\ hbkang@catholic.ac.kr
}

\begin{abstract}
In this paper, we propose a new multiple object tracking method via multi-layer multi-modal framework. To handle erroneous merge and labeling problem in multiple object tracking, we use a multi layer representation of dynamic Bayesian network and modified sampling method. For robust visual tracking, our dynamic Bayesian network based tracker fuses multi-modal features such as color and edge orientation histogram. The proposed method was evaluated under several real situations and promising results were obtained.
\end{abstract}

\section{Introduction}

Visual tracking in complex environment is an important task for surveillance, teleconferencing, and human computer interaction. It should be computationally efficient and robust to occlusion, changes in 3D pose and scale as well as distractions from background clutter. In particular, multiple object tracking of similar objects fails in the cases of adjacency or occlusion of tracked objects. For multiple identical or similar object tracking, it is necessary to solve merge and labeling problem correctly.

There have been some research works on multiple object tracking. In the multiple object tracking, it is very important to model the interaction among objects and solve the data association problem [1-6]. Usually, a joint state representation is used and joint data associations are inferred from the possible interactions between objects and observations. This approach requires a high computational cost due to the complexity of joint state representation. Yu et al. [4] propose collaboration approach among filters by modeling objects' joint prior using a Markov Random field. However, this approach has some limitations in correctly labeling objects. Qu et al. [5] suggest an interactively distributed multi-object tracking using a magnetic-inertia potential model. This approach is good for solving the labeling problem in identical multiple object tracking. However, the tracking performance suffers when the occlusion duration among objects gets a little longer.

For our multiple object tracking, we adopt a dynamic Bayesian network which has multi-layer representation and multi-modal features fusion. Dynamic Bayesian network (DBN) provides a unified probabilistic framework in integrating multimodalities by using a graphical representation of the dynamic systems. The proposed tracker has the following characteristics. First, multiple modalities are integrated in the dynamic Bayesian network to evaluate the posterior of each feature such as color 
and edge orientation. Secondly, the erroneous merge and labeling problem can be solved in two phases of DBN framework.

The paper is organized as follows. Section 2 discusses a multi-layer representation of dynamic Bayesian network. Section 3 presents our proposed multi-modal multiple object tracking method in DBN framework. Section 4 shows experimental results of our proposed method.

\section{Multi-layer Representation of DBN}

The Dynamic Bayesian Network (DBN) provides a coherent and unified probabilistic framework to determine the target object state in each frame by integrating modalities such as the prior model of reference state and evidence in target object candidate [7]. To construct DBN for visual tracking, we must specify three kinds of information such as the prior distribution over state variables $p\left(x_{0}\right)$, the transition model $p\left(x_{n} \mid x_{n-1}\right)$ and the observation model $p\left(y_{n} \mid x_{n}\right)$. The transition model $p\left(x_{n} \mid x_{n-1}\right)$ describes how the state evolves over time. The observation model $p\left(y_{n} \mid x_{n}\right)$ describes how the evidence variables are affected by the actual state of the object tracking. The target object candidate is evaluated by the posterior probability through the integration of multiple cues in DBN.

$$
p\left(x_{n} \mid y_{n}, x_{n-1}\right)
$$

where $x_{n}$ and $x_{n-1}$ are the target object candidate and reference object state, respectively and $y_{n}$ is the evidence of low-level features such as color and edge information from the target object candidate. In our visual tracking, we use two features such as color and edge orientation information. For evidence variables in our framework, we use color likelihood $p\left(c_{n} \mid x_{n}\right)$ and edge orientation likelihood $p\left(e_{n} \mid x_{n}\right)$ where $c_{n}$ and $e_{n}$ are the color and edge likelihood measurements at time $n$, respectively. The posterior probability like Eq. (1) is interpreted as

$$
p\left(x_{n} \mid c_{n}, e_{n}, x_{n-1}\right) \propto p\left(c_{n} \mid x_{n}\right) p\left(e_{n} \mid x_{n}\right) p\left(x_{n} \mid x_{n-1}\right)
$$

To deal with multiple object tracking, we denote the state of an individual object by $x_{n}^{i}$, where $i=1 \ldots \ldots . m$ is the index of objects and $n$ is the time index. To represent the interactive objects, we use a multi layer representation of DBN which is similar to hierarchical Hidden Markov Model. This is shown in Fig. 1. The top layer in DBN computes interactivity among hidden nodes by estimating the distance between a pair of objects. If the objects are adjacent or occluded, the interactivity among hidden nodes is computed. Using color and edge orientation likelihood information for the target object and the reference object, we classify the interactivities between two objects $\mathrm{A}$ and $\mathrm{B}$ into 5 cases such as " $\mathrm{A}$ and $\mathrm{B}$ are located near by" $(\mathrm{A}, \mathrm{B})$, "A is partially occluded by $B$ " $(A \subset B)$, "B is partially occluded by $A$ " $(B \subset A)$, "A is fully occluded by B "(B), and "B is fully occluded by A"(A). Based on objects' interactivities, the labeling node shown as a rectangle is activated or deactivated for each object state. After that, correct labels are assigned to each object state. 


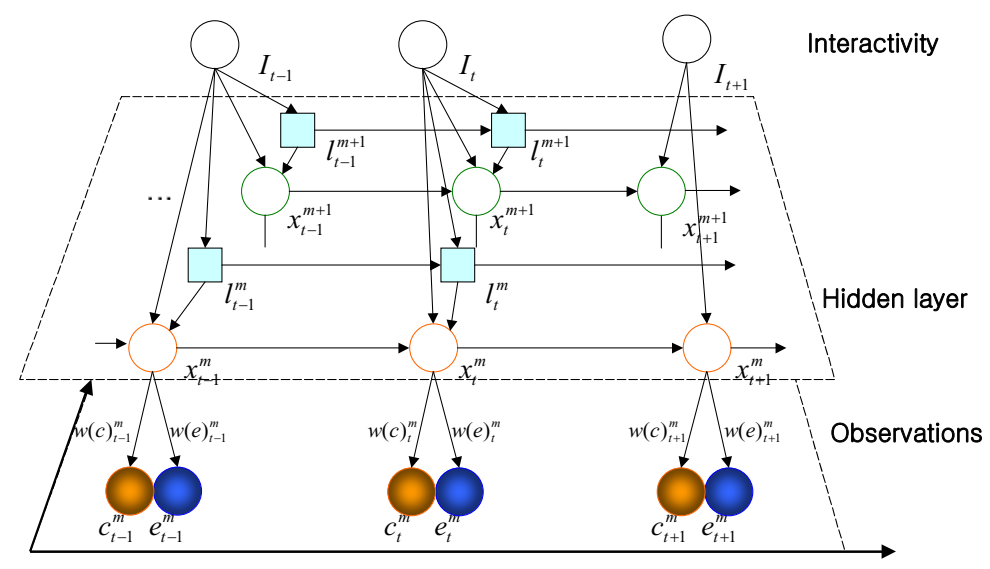

Fig. 1. Multi-layer multi-modal framework

\section{Multiple Object Tracking}

For multiple object tracking, it is necessary to solve erroneous merge problem where the tracker loses its target object and falsely coalesces with other trackers, and labeling problem where incorrect labels are assigned to the objects after occlusion.

\subsection{Erroneous Merge Problem}

To solve the erroneous merge problem, the accurate object state should be estimated when merge or split occurs. If two objects are merged (adjacent or partially occluded), it is very difficult to estimate exact position of the target object using conventional particle filtering because two objects are similar to each other. To reduce the effect of other object's presence, we use a Gaussian-weighted circular window in computing particle weight. For example, samples that are further away form the point having highest similarity value can be assigned smaller weights by employing a weighting function

$$
\varphi(r)=\left\{\begin{array}{c}
1-r^{2}: r<1 \\
0: \text { otherwise }
\end{array}\right.
$$

where $r$ is the normalized distance from the highest similar point to the sample. Fig. 2(a) shows the original similarity distribution of samples for red object. By using weighting function like Eq. (3), the similarity distribution is transformed to that in Fig. 2(b). So, we can estimate exact target object position regardless of the green object in Fig. 2.

When the objects are merged, the tracker for the occluded object computes the similarity distribution of the reference model. If the similarity value is larger than the threshold value, weighting function like Eq. (3) is used to estimating the position of that object. Then, the tracker can solve the problem of false coalescence. 


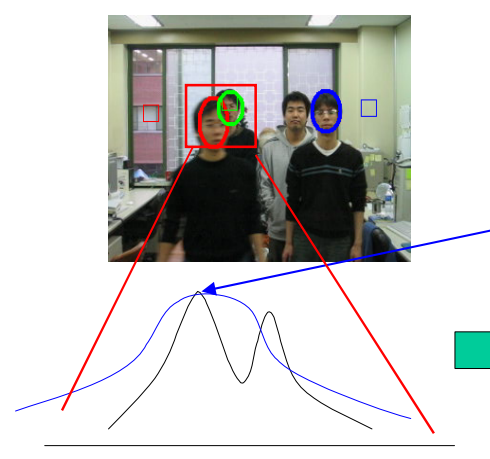

(a)
The point having highest similarity for red object

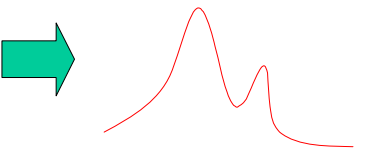

(b)

Fig. 2. (a) Original similarity distribution of samples for red object, (b) Transformed similarity distribution

\subsection{Labeling Problem}

To correctly assign a label to target object after occlusion, it is necessary to robustly discriminate target object candidates. We use multi-modal features such as color and edge orientation histogram.

The color likelihood $p\left(c_{n} \mid x_{n}\right)$ is defined as

$$
p\left(c_{n} \mid x_{n}\right)=\left[\frac{1}{\sqrt{2 \pi \sigma}} e^{-\frac{1-\sum_{u=1}^{m} \sqrt{p_{i}^{(u)} q^{(u)}}}{2 \sigma^{2}}}\right]
$$

where $p_{i}^{(u)}$ is the $i^{\text {th }}$ object candidate's color distribution and $q^{(u)}$ is color distribution of reference object.

The edge likelihood is computed from edge orientation histogram similar to SIFT [8]. To compute edge orientation, we detect edges using horizontal and vertical Sobel operators. After computing the strength and orientation of the edges, we apply threshold operation to remove outliers. The edge intensity and orientation of the target object is quantized into 8 bins ( 0 degree, 45 degree...) and displayed in each quarter plane. Peaks in the orientation histogram correspond to dominant directions of local gradients. After detecting the dominant orientation, edge descriptor for the reference model will be represented relative to this dominant direction and therefore achieve invariance to image rotation. We compute $16 \mathrm{x} 16$ edge descriptor for the center point of the target object candidate. Fig. 3 shows the edge descriptor for a quarter plane of a target candidate. The edge likelihood between the target candidate and the reference model is computed as L2 distance between two edge orientation histograms. The edge likelihood $p\left(e_{n} \mid x_{n}\right)$ is defined as 


$$
p\left(e_{n} \mid x_{n}\right)=\left[\frac{1}{\sqrt{2 \pi \sigma}} e^{-\frac{\rho^{2}\left(p_{i}^{(u)} q^{(u)}\right)}{2 \sigma^{2}}}\right]
$$

where $\rho(p, q)$ is Euclidean distance, $p_{i}^{(u)}$ is the $i^{\text {th }}$ object candidate's edge histogram distribution and $q^{(u)}$ is edge histogram distribution of reference object. Our edge likelihood is invariant to rotation and is discriminative against the background with confusing colors.

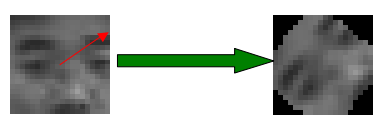

Rotation by dominant orientation

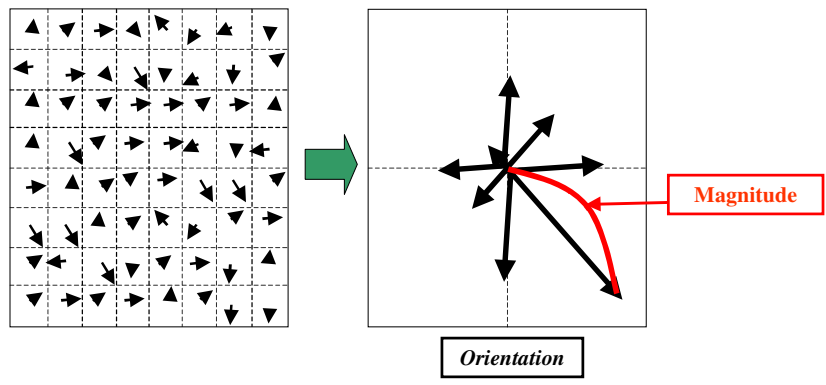

Fig. 3. Edge Orientation Histogram in quarter plane

The confidence weights for multi-cues such as color and edge likelihood are conditionally determined by previous weights. The observation likelihood is decided by each cue's weight. By adopting adaptive confidence weights, our tracker can discriminate target objects and then the correct label will be assigned to the target object.

For the approximate inference in DBN, we use the modified particle filtering since it seems to maintain a good approximation to the true posterior by using a constant number of samples [9]. In our proposed approach, the sampling-based method is executed as follows:

Step 1: $N$ samples are created by sampling from the prior distribution at time 0.

Step 2: Each sample is propagated forward by sampling from the transition model like $p\left(x_{n}^{i} \mid x_{n-1}^{i}\right)$.

Step 3:Each sample is weighted by the log likelihood such as $k_{1} p\left(c_{n}^{i} \mid x_{n}^{i}\right)+k_{2} p\left(e_{n}^{i} \mid x_{n}^{i}\right)$ where $k_{1}, k_{2}$ are the confidence weight of the likelihood of each sample. When the objects are adjacent or occluded, the log likelihood is $k_{1} p\left(c_{n}^{i} \mid x_{n}^{i}\right) \varphi()+k_{2} p\left(e_{n}^{i} \mid x_{n}^{i}\right) \varphi()$, where $\varphi()$ is the Gaussian weighting function.

Step 4: The population is re-sampled to generate a new population of $N$ samples with weighted-sample-with-replacement. 


\section{Experimental Results}

Our proposed DBN-based visual tracking algorithm is implemented on a P4-3.0Ghz system with $320 \times 240$ image size. The number of particles is 400 . The target object size is 16 x 16 pixels. For the implementation of the multi-layer DBN, we used Intel's Probabilistic Network Library (OpenPNL) [10] for building blocks. The input for $\mathrm{DBN}$ is the weighted sum of color and edge orientation likelihood.

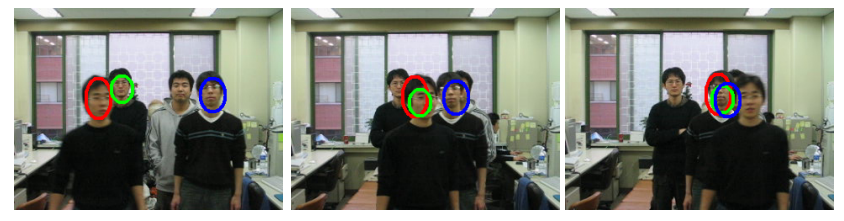

(a)
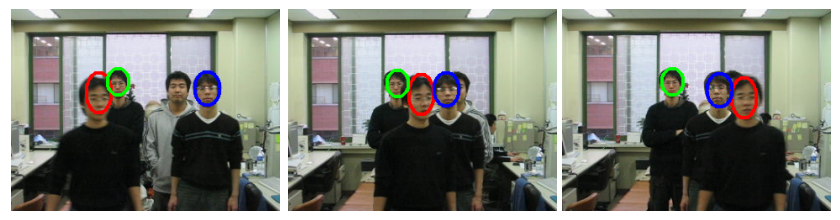

(b)

Fig. 4. (a) Conventional Particle Filtering, (b) Proposed Method
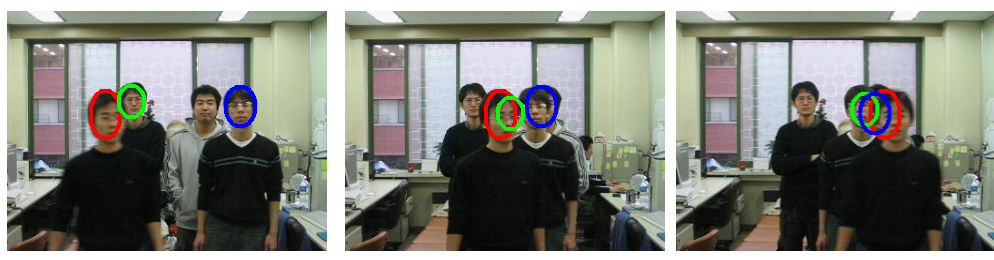

(a)
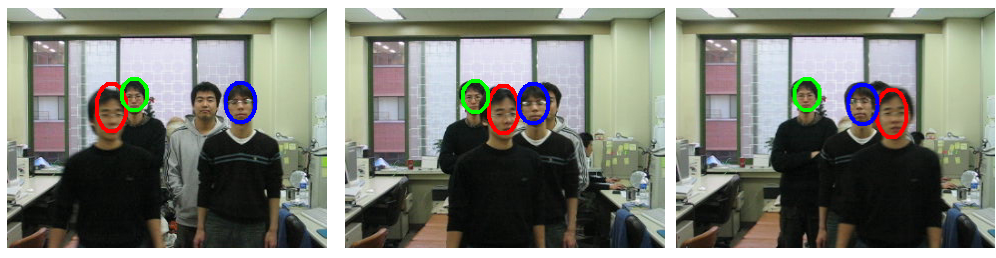

(b)

Fig. 5. (a) Color-based tracking, (b) Color and edge-based tracking 


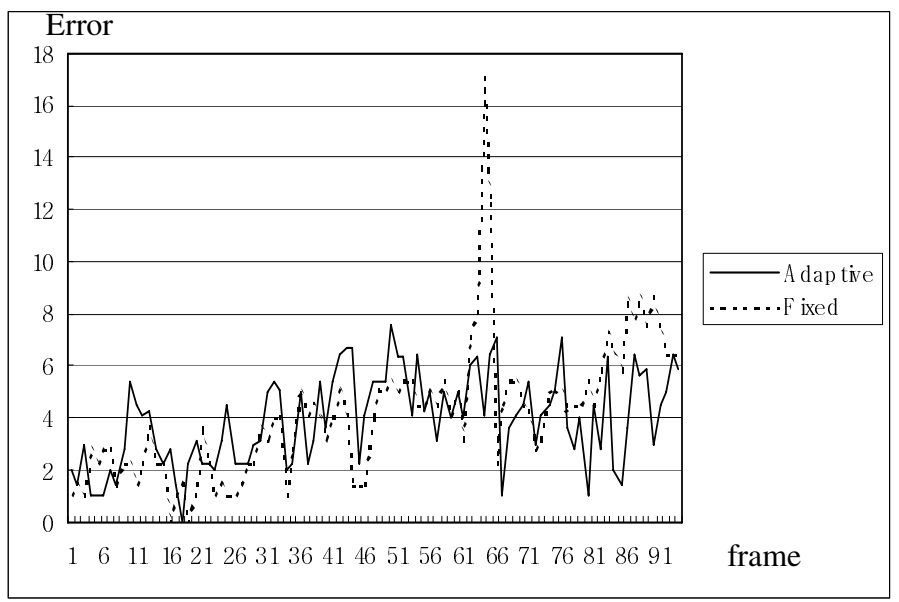

Fig 6. Tracking errors using fixed and adaptive confidence weights for color and edge likelihood

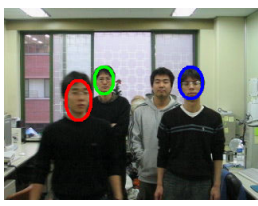

\#28

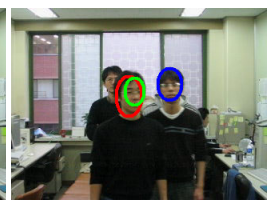

\#36

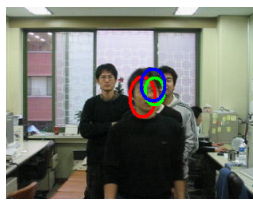

\#44

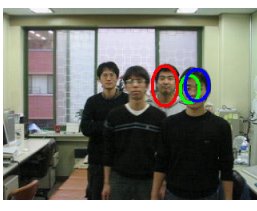

\#55

(a)

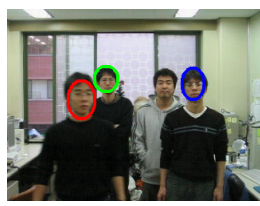

\#28

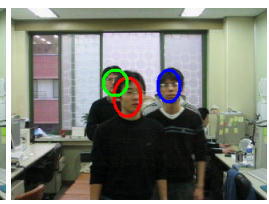

\#36

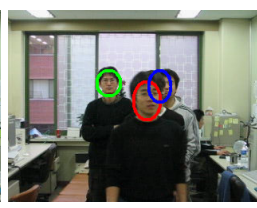

\#44

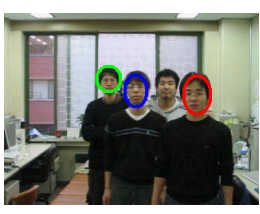

\#55

(b)

Fig. 7. Comparison of IDMOT[5] and our proposed tracker for office sequence (a) IDMOT result, (b) proposed tracker result

We made several experiments in a variety of environments to show the robustness of our proposed method. The first video sequence contains four moving persons in the office. This sequence is very difficult for multiple object tracking due to frequent occlusion. Fig. 4 shows the result of conventional particle filtering and our proposed modified particle filtering using Gaussian-weighted circular window. Our proposed method estimates accurate position of the target object. 


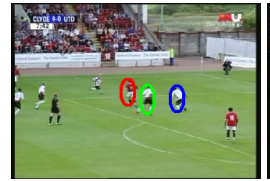

\#31

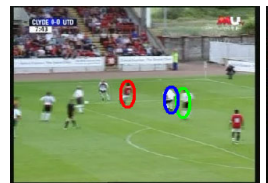

\#47

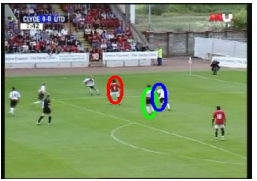

\#37

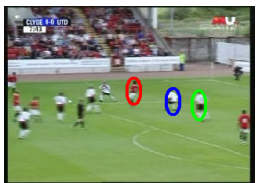

\#51

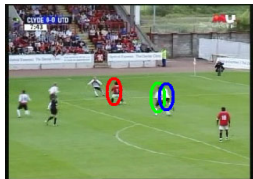

\#40

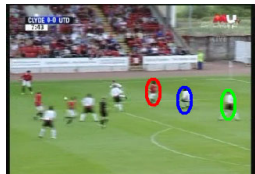

\#56

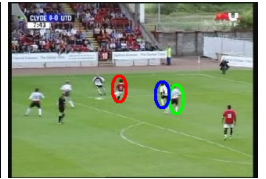

\#44

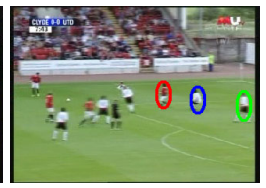

\#58

Fig. 8. Results of the proposed multi-modal multi-object tracker for the soccer game sequence

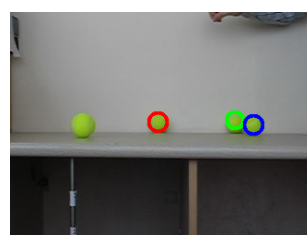

\#20

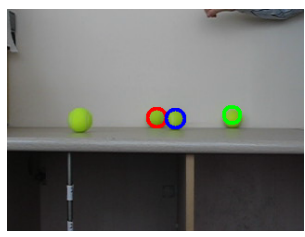

\#40

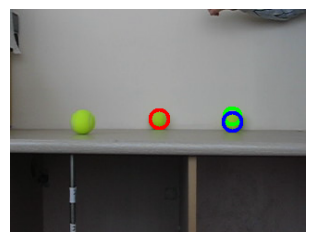

\#24

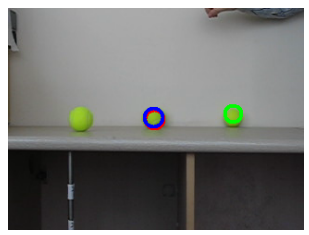

\#43

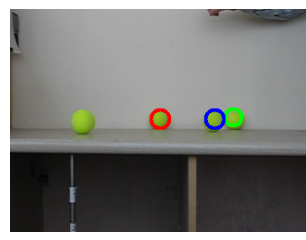

\#32

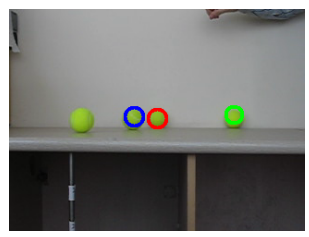

\#49

Fig. 9. Tracking result of tennis ball sequence

We experimented multi-cues approach in multiple object tracking. Fig. 5(a) shows the result of color only tracking and Fig. 5(b) shows the tracking result using our color and edge orientation likelihood. The confidence weights in color and edge likelihood are also important in robust tracking. Fig. 6 shows the errors occurred in tracking that used the fixed and adaptive confidence weights in our proposed method.

In Fig. 7, we compared our proposed method with other tracking method. IDMOT [5] suffers from labeling problems in long duration of occlusion (see Fig. 7(a)). However, our approach performs well solving both erroneous merge and labeling problem. This is shown in Fig. 7(b).

The second video sequence is the soccer game sequence. Each object moves independently. The image size is $320 \times 240$. Examples of the tracking results are shown in Fig. 8. Our proposed algorithm successfully tracked all objects throughout all frames. Fig. 9 shows the tracking result of tennis ball sequence. The labels in each ball are correct. 


\section{Conclusions}

In this paper, multi layer representation of dynamic Bayesian network is proposed for multiple object tracking. For robust tracking, we implement a modified sampling and multi-modal tracking method that integrates color and edge orientation histogram. Our proposed tracker can handle erroneous merge and labeling problem in multiple object tracking. We have presented results from realistic scenarios to show the validity of the proposed approach. Compared to other tracking algorithms, our proposed system shows better and more robust tracking performance.

\section{Acknowledgements}

This work was supported by the Culture Research Center Project, the Ministry of Culture \& Tourism and the KOCCA R\&D program in Korea.

\section{References}

1. Mccormick, J., Blake, A.: A probabilistic exclusion principle for tracking multiple objects. Int. J. Comput. Vis (2000)

2. Isard, M., Mccormick, J.: Bramble: A Bayesian multiple blob tracker. In: Proc. ICCV 01 (2001)

3. Khan, Z., Balch, T., Dellaert, T.: An MCMC-based particle filter for tracking multiple interacting targets. In: Pajdla, T., Matas, J(G.) (eds.) ECCV 2004. LNCS, vol. 3021, Springer, Heidelberg (2004)

4. Yu, T., Wu, T.: Collaborative Tracking of Multiple Targets. In: Proc. CVPR'04 (2004)

5. Qu, W., Schonfeld, D., Mohamed, M.: Real-time Interactively Distributed Multi-Object Tracking Using a Magnetic-Inertia Potential Model. In: Proc. ICCV'05 (2005)

6. Yang, C., Duraiswami, R., Davis, R.: Fast Multiple Object Tracking via a Hierarchical Particle Filter. In: Proc. ICCV'05 (2005)

7. Kang, H-.B., Cho, S.: A Dynamic Bayesian Network-based Framework for Visual Tracking. In: Blanc-Talon, J., Philips, W., Popescu, D.C., Scheunders, P. (eds.) ACIVS 2005. LNCS, vol. 3708, pp. 603-610. Springer, Heidelberg (2005)

8. Lowe, D.: Distinctive image features from scale-invariant keypoints. Int'l J. Computer Vision, 91-110 (2004)

9. Nummiaro, K., Koller-Meier, E., Van Gool, L.: A Color-Based Particle Filter, First International Workshop on Generative-Model-Based Vision pp. 53-60 (2002)

10. Intel Open Source Probabilistic Network Library (OpnePNL) http://www.intel.com/research/mrl/pnl 